1. S. Chandrasekhar \& D. Elbent, "The roots of $Y_{n}(\lambda \eta) J_{n}(\lambda)-J_{n}(\lambda \eta) Y_{n}(\lambda)=0$," Proc. Cambridge Philos. Soc., v. 50, 1954, pp. 266-268. MR 15, 744.

2. H. E. Fetris \& J. C. Cassin, "An extended table of zeros of cross products of Bessel functions," Rept. No. ARL 66-0023, Office of Aerospace Research, U. S. Air Force, WrightPatterson Air Force Base, Ohio.

3. A. Fletcher, J. C. P. Miller, L. Rosenhead \& L. J. Comrie, An Index of Mathematical Tables, 2nd ed., Addison-Wesley, Reading, Mass., 1962. MR 26 \#365a, b.

4. J. McMahon, "On the roots of the Bessel and certain related functions," Ann. of Math., v. 9,1894 , pp. $23-30$.

5. F. W. J. Olver (Editor), Bessel Functions. Part III; Zeros and Associated Values, Royal Society Mathematical Tables, Vol. 7, Cambridge Univ. Press, New York, 1960, Table I, pp. 2-14. MIR $22 \# 10202$.

\title{
More on the Calculation of the Integral
}

$$
I_{n}(b)=\frac{2}{\pi} \int_{0}^{\infty}\left(\frac{\sin x}{x}\right)^{n} \cos b x d x
$$

\section{By Henry E. Fettis}

The evaluation of this integral has been the subject of two recent papers [1], [2]. Although the integral can be expressed in a simple analytical form, namely

$$
\begin{array}{rlrl}
\left\{I_{n}(b)\right. & \left.=\frac{n}{2^{n-1}} \sum_{k=1:}^{\left[(n-b)^{\prime 2}\right.} \frac{(-)^{k}(n-b-2 k)^{n-1}}{k !(n-k) !}, b<n\right\}, \\
& =0, & b \geqq n,
\end{array}
$$

(where $[(n-b) / 2\}$ denotes the largest integer less than $(n-b) / 2)$, the use of the above expression for large $n$ has not proved satisfactory. Alternative schemes in lieu of (1) have been proposed by Medhurst and Roberts [1] and Thompson [2]. These essentially are recursive-type methods, in which results for higher values of $n$ and $b$ are computed from starting values obtained for lower order and argument by the exact expression (1). Such schemes have the disadvantage that the direct computation for a given $n$ and $b$ is not possible. The present paper proposes a method which overcomes this difficulty and allows the integral to be computed directly. The formulae work equally well for small and large values of $b$, and are particularly well suited to computation for moderate and large $n$.

The basis of the present method is the Poisson summation formula [3]. In its most general form it may be written as follows

$$
\sum_{k=-\infty}^{\infty} \exp \left[i k u_{1}\right] f(a+k d)=\frac{1}{d} \sum_{m=-\infty}^{\infty} G\left(\frac{2 \pi m+\imath}{d}\right) \exp \left[-i(a / d)\left(2 \pi m+u_{1}\right)\right]
$$

where $G$ is the Fourier transform of $f$, namely

Received May 18, 1967. 


$$
G(y)=\int_{-\infty}^{\infty} f(x) e^{i x y} d x
$$

The formula (2) may be applied to the present problem by taking $a=0, f(x)=$ $(\sin x / x)^{n}$. We find that

$$
G(y)=\pi I_{n}(y) .
$$

Setting $u_{1} / d=t$, and noting that $f(x)$ is an even function, we get

$$
\begin{gathered}
I_{n}(t)+I_{n}(t+2 \pi / d)+I_{n}(t-2 \pi / d)+I_{n}(t+4 \pi / d)+I_{n}(t-4 \pi / d)+\cdots+ \\
5) \quad=\frac{d}{\pi}\left[1+2 \cos (d t)\left(\frac{\sin d}{d}\right)^{n}+2 \cos (2 d t)\left(\frac{\sin 2 d}{2 d}\right)^{n}+\cdots+\right] .
\end{gathered}
$$

We now specialize Eq. (5) in two ways. First we set $t=0$ and $d=2 \pi / b$, and obtain

$$
\begin{aligned}
& I_{n}(0)+2 I_{n}(b)+2 I_{n}(2 b)+\cdots+ \\
& \quad=\frac{2}{b}\left[1+2\left(\frac{\sin (2 \pi / b)}{2 \pi / b}\right)^{n}+2\left(\frac{\sin (4 \pi / b)}{4 \pi / b}\right)^{n}+\cdots+\right] .
\end{aligned}
$$

Next, we set $t=b$ and $d=2 \pi / n$. This gives

$$
\begin{aligned}
& I_{n}(b)+I_{n}(n+b)+I_{n}(n-b)+I_{n}(2 n+b)+I_{n}(2 n-b)+\cdots+ \\
& \frac{2}{\pi}=\left[1+2 \cos \frac{2 \pi b}{n}\left(\frac{\sin (2 \pi / n)}{2 \pi / n}\right)^{n}+2 \cos \frac{4 \pi b}{n}\left(\frac{\sin (4 \pi / n)}{4 \pi / n}\right)^{n}+\cdots+\right] .
\end{aligned}
$$

In particular, if $b>n / 2$, then $I_{n}(k b)=0$ for $k>1$, and Eq. (6) becomes

(8) $I_{n}(b)=\frac{1}{b}\left[1+2\left(\frac{\sin (2 \pi / b)}{2 \pi / b}\right)^{n}+2\left(\frac{\sin (4 \pi / b)}{4 \pi / b}\right)^{n}+\cdots+\right]-\frac{1}{2} I_{n}(0)$.

For $b=n$, we obtain Butler's result [4], namely

$$
I_{n}(0)=\frac{2}{n}\left[1+2\left(\frac{\sin (2 \pi / n)}{2 \pi / n}\right)^{n}+2\left(\frac{\sin (4 \pi / n)}{4 \pi / n}\right)^{n}+\cdots+\right] .
$$

Next suppose $b<n / 2$. Then $n-b>n / 2$, and $n k \pm b>n(1+k) / 2>n$ for $k>1$. Hence (7) gives

$$
\begin{aligned}
I_{n}(b)+I_{n}(n-b)=\frac{2}{n}[1+ & 2 \cos \left(\frac{2 \pi b}{n}\right)\left(\frac{\sin (2 \pi / n)}{2 \pi / n}\right)^{n}+\cdots \\
& \left.\cdots+2 \cos \left(\frac{4 \pi b}{n}\right)\left(\frac{\sin (4 \pi / n)}{4 \pi / n}\right)^{n}+\cdots+\right] .
\end{aligned}
$$

For $b=n / 2$ both (8) and (10) give

$$
I_{n}\left(\frac{n}{2}\right)=\frac{1}{n}\left[1-2\left(\frac{\sin (2 \pi / n)}{2 \pi / n}\right)^{n}+2\left(\frac{\sin (4 \pi / n)}{4 \pi / n}\right)^{n}+\cdots+\right] .
$$

Formulae (8) and (10) have the advantage that convergence is virtually unaffected by the value of " $b$ ". Further it is easy to predict in advance the number of terms needed for a prescribed accuracy by examining the magnitude of the successive maxima of $|\sin \theta / \theta|$. These maxima occur approximately at the points $\theta=$ 
$(2 k+1)(\pi / 2)$ where $k=1,2, \cdots$. Their magnitude, for all practical purposes can be estimated as $1 /\left(k+\frac{1}{2}\right)$, and the contribution in the interval $[k \pi,(k+1) \pi]$ as $2\left[k+\frac{1}{2}\right]^{-n}$, so that for $n>10$ the summation can be terminated after three or less cycles of the integrand have been covered.

A formula similar to (6) can be obtained by taking $a=\pi / b, d=2 \pi / b, u=0$ in Eq. (2).* This gives

$$
I_{n}(0)-2 I_{n}(b)+2 I_{n}(2 b)-\cdots+=\frac{4}{b} \sum_{k=0}^{\infty}\left\{\frac{\sin \left(k+\frac{1}{2}\right)(2 \pi / b)}{\left(k+\frac{1}{2}\right)(2 \pi / b)}\right\}^{n} .
$$

For $b>n / 2$ the left side reduces to $I_{n}(0)-2 I_{n}(b)$, so that

$$
I_{n}(b)=\frac{1}{2} I_{n}(0)-\frac{2}{b} \sum_{k=0}^{\infty}\left\{\frac{\sin \left(k+\frac{1}{2}\right)(2 \pi / b)}{\left(k+\frac{1}{2}\right)(2 \pi / b)}\right\}^{n} .
$$

Combining this with (8) and changing the index of summation, we obtain a formula for $I_{n}(b)$ which is free of $I_{n}(0)$ :

$$
I_{n}(b)=\frac{1}{2 b}\left[1+2 \sum_{m=1}^{\infty}(-)^{m}\left(\frac{\sin (m \pi / b)}{(m \pi / b)}\right)^{n}\right], \quad b>n / 2,
$$

and by setting $b=n$ we obtain another expression for $I_{n}(0)$ :

$$
I_{n}(0)=\frac{4}{b} \sum_{k=0}^{\infty}\left\{\frac{\sin \left(k+\frac{1}{2}\right)(2 \pi / n)}{\left(k+\frac{1}{2}\right)(2 \pi / n)}\right\}^{n} .
$$

Sample calculations of $I_{n}(b)$ for $n=12, b=0,4,6,8$

$[\sin (2 k \pi / n) / 2 k \pi / n]^{n} \quad[\sin (2 k \pi / b) / 2 k \pi / b]^{n} \quad \cos 2 k \pi b / n[\sin (2 k \pi / n) / 2 k \pi / n]^{n}$

$$
k
$$

$$
(b=8) \quad \cos (2 k \pi b / n) \quad(b=4)
$$

$1 \quad .57498$

50916

$.28362 \quad 30021$

$-.5-.28749$

25458

$2 \quad .10233$

49931

.00443

16094

$-.5$

$-.05116$

74966

$3 \quad .00443$

16094

.00000

05337

1.0 .00443 16094

$4 \quad .00002 \quad 49841$

$.00000 \quad 00000$

$-.5 \quad-.00001$

24920

$5 \quad .00000$

00024

$.00000 \quad 00012$

$-.5$

$-.00000$

00012

$\begin{array}{lll}6 & .00000 & 00000\end{array}$

$.00000 \quad 00083$

$\begin{array}{lll}1.0 & .00000 & 00000\end{array}$

$\begin{array}{lll}7 & .00000 & 00000\end{array}$

$-.5$

00000

00000

$\begin{array}{lll}8 & 00000 & 00061\end{array}$

$-.5$

$-.00000$

00031

$9 \quad 00000 \quad 00083$

$\begin{array}{ll}1.0 & .00000\end{array}$

00083

$\begin{array}{lll}10 & 00000 & 00004\end{array}$

$-.5-.00000$

00002

$$
\begin{aligned}
.5+\sum\left[\frac{\sin (2 k \pi / n)}{2 k \pi / n}\right]^{n}=1.1817766955 ; & I_{12}(0)=.3939255652 \\
.5+\sum(-)^{k}\left[\frac{\sin (2 k \pi / n)}{2 k \pi / n}\right]^{n}=-.4770567278 ; & I_{12}(6)=.0038238787 \\
.5+\sum\left[\frac{\sin (2 k \pi / b)}{2 k \pi / b}\right]^{n}=.7880551547 ; & I_{12}(8)=.0000510061
\end{aligned}
$$

$.5+\sum \cos 2 k \pi b / n\left[\frac{\sin (2 k \pi / n)}{2 k \pi / n}\right]^{n}=.1657590791 ; \quad I_{12}(4)=.0552020202$

* The author is indebted to the referee for this additional material. 
Applied Mathematics Research Laboratories Wright-Patterson Air Force Base, Ohio

1. R. G. Medhurst \& J. H. Roberts, "Evaluation of the integral $I_{n}(b)=(2 / \pi) \int_{0}^{\infty}((\sin x) / x)^{n}$ $\cos (b x) d x$," Math. Comp., v. 19, 1965, pp. 113-117. MR 30 \#2665.

2. R. Thompson, "Evaluation of $I_{n}(b)=(2 / \pi) \int_{0}^{\infty}((\sin x) / x)^{n} \cos (b x) d x$, , Math. Comp., v. 20, 1966, pp. 330-331. MR 33 \#859.

3. W. Magnus \& F. Oberhetringer, Formeln und Sätze für die speziellen Funktionen der mathematischen Physik, 2nd ed., Springer-Verlag, Berlin, 1948, p. 217; English transl., Chelsea, New York, 1949. MR 10, 38; MR 10, 532.

4. R. Butler, "On the evaluation $\int_{0}^{\infty}\left(\sin ^{m} t\right) / t^{m} d t$ by the trapezoidal rule," Amer. Math. Monthly, v. 67, 1960, pp. 566-569. MR $22 \# 4841$. 